\title{
Effects of air pollution and habitual exercise on the risk of death: a longitudinal cohort study
}

\author{
Cui Guo PhD, Tsung Yu PhD, Ly-yun Chang PhD, Changqing Lin PhD, Hsiao Ting Yang MSc, Yacong Bo PhD, \\ Yiqian Zeng MPH, Tony Tam PhD, Alexis K.H. Lau PhD, Xiang Qian Lao PhD
}

Cite as: CMAJ 2021 August 16;193:E1240-9. doi: 10.1503/cmaj.202729

See related article at www.cmaj.ca/lookup/doi/10.1503/cmaj.211282

\begin{abstract}
Background: Exercise may exacerbate the adverse health effects of air pollution by increasing the inhalation of air pollutants. We investigated the combined effects of long-term exposure to fine particle matter $\left(\mathrm{PM}_{2.5}\right)$ and habitual exercise on deaths from natural causes in Taiwan.
\end{abstract}

Methods: We recruited 384130 adults (aged $\geq 18 \mathrm{yr}$ ) with 842394 medical examination records between 2001 and 2016, and followed all participants until May 31 , 2019. We obtained vital data from the National Death Registry of Taiwan. We estimated $\mathrm{PM}_{2.5}$ exposure using a satellitebased spatiotemporal model, and collected information on exercise habits using a standard self-administered questionnaire. We analyzed the data using a Cox regression model with timedependent covariates.

Results: A higher level of habitual exercise was associated with a lower risk of death from natural causes, compared with inactivity (hazard ratio [HR] 0.84, 95\% confidence interval $[\mathrm{Cl}] 0.80-0.88$ for the moderate exercise group; HR 0.65 , $95 \% \mathrm{Cl} 0.62-0.68$ for the high exercise groups), whereas a higher $\mathrm{PM}_{2.5}$ exposure was associated with a higher risk of death from natural causes compared with lower exposure (HR 1.02, 95\% Cl 0.98-1.07, and HR $1.15,95 \% \mathrm{Cl} 1.10-1.20$, for the moderate and high $\mathrm{PM}_{2.5}$ exposure groups, respectively). Compared with inactive adults with high $\mathrm{PM}_{2.5}$ exposure, adults with high levels of habitual exercise and low $\mathrm{PM}_{2.5}$ exposure had a substantially lower risk of death from natural causes. We found a minor, but statistically significant, interaction effect between exercise and $\mathrm{PM}_{2.5}$ exposure on risk of death (HR 1.03 95\% Cl 1.01-1.06). Subgroup analyses, stratified by $\mathrm{PM}_{2.5}$ categories, suggested that moderate and high levels of exercise were associated with a lower risk of death in each $\mathrm{PM}_{2.5}$ stratum, compared with inactivity.

Interpretation: Increased levels of exercise and reduced $\mathrm{PM}_{2.5}$ exposure are associated with a lower risk of death from natural causes. Habitual exercise can reduce risk regardless of the levels of $\mathrm{PM}_{2.5}$ exposure. Our results suggest that exercise is a safe health improvement strategy, even for people residing in relatively polluted regions.
A ir pollution and physical inactivity are both major public health challenges worldwide. ${ }^{1}$ Air pollution was the fifth leading cause of disability related to health and accounted for 4.9 million deaths worldwide in $2017 . .^{2}$ More than $91 \%$ of the global population lives in areas where air quality does not meet the World Health Organization (WHO) guidelines. ${ }^{3}$ In addition, physical inactivity was the fourth leading risk factor for death globally, accounting for 5.3 million deaths worldwide in 2012. ${ }^{4}$ The WHO has challenged its member states to reduce physical inactivity by $15 \%$ by $2030 . .^{5}$

As people exercise, their ventilation rate increases, which increases the volume of air pollutants they inhale. This may exacerbate the adverse health effects of air pollutants. Thus, the risk-benefit relation between air pollution and exercise needs to be assessed to understand whether it is safe to exercise regularly in polluted regions. Indeed, some studies have shown that acute exposure to air pollution when exercising may override the benefits of exercise. ${ }^{6,7}$ It is possible that the effects of long-term exposure to air pollution may be irreversible and cause a much larger disease burden than short-term exposure. Limited information exists on the combined effects of long-term exposure to air pollution and habitual exercise on human health, and findings have been inconsistent depending on health outcome. Three cohort studies have explored the relation between air pollution, physical activity and risk of death in Hong Kong, ${ }^{8}$ Denmark and the United States, ${ }^{9}$ with relatively small sample sizes. ${ }^{10}$ Therefore, we sought to investigate the combined effects of habitual exercise and long-term exposure to fine particle matter $\left(\mathrm{PM}_{2.5}\right)$ on the risk of death from natural causes (i.e., deaths not attributable to accident, suicide or homicide) using a longitudinal cohort of adults in Taiwan, where the annual $\mathrm{PM}_{2.5}$ concentrations are 1.6 times higher than the WHO-recommended limit. We hypothesized that 
the beneficial effects of habitual exercise on risk of death may outweigh the risk of high levels of air pollutants inhaled during exercise.

\section{Methods}

\section{Study design and setting}

We conducted our study in Taiwan, which has a tropical monsoon climate in the south and subtropical monsoon climate in the north and an annual average temperature of $22^{\circ} \mathrm{C} .{ }^{11}$ Participants were part of an ongoing open cohort, for which details have been described in our previous publications. ${ }^{12-14}$ In brief, the MJ Health Management Institution has been providing residents of Taiwan with a standard medical screening program since 1994. Participants join the program through a paid membership and are encouraged to visit the institution periodically. During each medical visit, participants receive a series of medical examinations, including anthropometric measurements, spirometry tests, blood and urinary tests, and imaging tests. They also complete a standard, self-administered questionnaire.

Data from the medical examinations have been managed electronically since 1996. This cohort is an open, dynamic cohort without an end date for recruitment or follow-up. Each year, around 20000 new members are recruited to the cohort, in addition to revisits by existing members. More than 600000 residents have been recruited as of December 2016, with 1.5 million medical visits. Written informed consent is given by each participant before each medical examination.

\section{Participants}

We included adults ( $\geq 18 \mathrm{yr}$ ) who joined the medical screening program between 2001 and 2016, when $\mathrm{PM}_{2.5}$ concentration was available. We excluded participants with missing $\mathrm{PM}_{2.5}$ exposure because of incomplete addresses and participants with missing data. We followed participants from their entry date (i.e., the first medical examination) until May 31, 2019, or the date of death, if earlier.

\section{Exposures and outcomes}

We obtained information on vital status and causes of death from the National Death Registry, which is maintained by the Ministry of Health and Welfare of Taiwan. ${ }^{15}$ The main outcome was death from natural causes (International Classification of Diseases [ICD]-9 codes: 001-779; ICD-10 codes: A00-R99). Participants with an accidental cause of death were censored the time of death.

Details of the $\mathrm{PM}_{2.5}$ exposure assessment have been described previously. ${ }^{12,16,17}$ In brief, a spatiotemporal model was developed at a resolution of $1 \mathrm{~km}^{2}$ using aerosol optical depth data collected via the Moderate Resolution Imaging Spectroradiometer from the Terra and Aqua satellites, carried aboard the U.S. National Aeronautics and Space Administration's Earth Observing System. We obtained ground-level aerosol optical depth data from the aerosol robotic network in Taipei, Taiwan, to calibrate the data. Finally, the spatiotemporal model was validated by comparing the estimated $\mathrm{PM}_{2.5}$ concentration with the $\mathrm{PM}_{2.5}$ concentration from air pollution monitoring stations across Taiwan.
We assigned the estimated $\mathrm{PM}_{2.5}$ exposure to participants according to their geocoded addresses. We calculated long-term exposure to $\mathrm{PM}_{2.5}$ as the 2-year average concentration in the year of medical examination and in the previous year. We conducted our analysis using both continuous $\mathrm{PM}_{2.5}$ exposure data (per $10 \mathrm{\mu g} / \mathrm{m}^{3}$ ) and categorical $\mathrm{PM}_{2.5}$ exposure, whereby we grouped participants into 3 categories based on the tertile cut-off points (low: $<22.4 \mu \mathrm{g} / \mathrm{m}^{3}$, moderate: $22.4-26.0 \mu \mathrm{g} / \mathrm{m}^{3}$, high: $\geq 26.0 \mu \mathrm{g} / \mathrm{m}^{3}$ ).

We collected information on habitual exercise; the details have been described in our previous studies. ${ }^{13,18-20}$ Briefly, a standard self-administrated questionnaire was used to collect information on leisure time exercise. The questionnaire was validated by comparing exercise levels with data from the National Health Interview Survey, and a test-retest approach was used to assess its reliability. ${ }^{13}$ We obtained weekly data on the duration and intensity of habitual exercise in the month before each medical examination. We classified exercise intensity into 4 categories: light (e.g., walking), moderate (e.g., brisk walking), mediumvigorous (e.g., jogging), or high-vigorous (e.g., running). We assigned intensity categories a metabolic equivalent (MET) value of 2.5, 4.5, 6.5 and 8.5, respectively, where 1 MET equals $1 \mathrm{kcal} / \mathrm{h} / \mathrm{kg}$ bodyweight. ${ }^{13,21}$ If a participant did not undertake any exercise, then we assigned a MET value of 0 . For participants who reported activities in more than 1 intensity category, we weighted the MET value according to the time spent in each category. We calculated the exercise volume (MET-h) as the product of the intensity (MET) and duration (h). For analysis, we grouped participants into 3 categories based on the tertile cut-off points (i.e., inactive: 0 MET-h, moderate: 0 to 8.75 MET-h, high: > 8.75 MET-h).

\section{Covariates}

Participants' weight and height was measured when they were wearing light clothing and no shoes. An overnight fasting blood sample was collected in the morning, and information on demographics, lifestyles, physical activity at work and medical history was measured using a standard self-administered questionnaire.

We included the following covariates in the main analysis: age, sex, education (i.e., less than high school, completed high school, college or university, postgraduate), body mass index, physical labour at work (mostly sedentary, mostly standing or walking, hard labour), cigarette smoking (never, former, current), alcohol drinking (never/seldom, former, current), vegetable and fruit intake (seldom [ $<1$ serving/day], moderate [1-2 servings/day], frequent [ $>2$ servings/day]), occupational exposure to dust or solvents, season and year of enrolment.

Additional information on participant selection, outcome ascertainment, $\mathrm{PM}_{2.5}$ exposure assessment, evaluation of habitual exercise and covariates is presented in Appendix 1, Figure E1, available at www.cmaj.ca/lookup/doi/10.1503/cmaj.202729/ tab-related-content.

\section{Statistical analysis}

We used Cox regression models with time-dependent covariates to investigate the combined effects of $\mathrm{PM}_{2.5}$ exposure and habitual exercise on deaths from all natural causes. To control 
for clustering effects within the same city, we used a city-level random intercept. We developed 2 models to incrementally adjust for the covariates. Model 1 adjusted for age, sex and education, and Model 2 further adjusted for body mass index, physical labour at work, smoking status, alcohol consumption, vegetable and fruit intake, occupational exposure to dusts or solvents, season and year of cohort enrolment. We ran additional models that mutually adjusted for $\mathrm{PM}_{2.5}$ and exercise for comparison (i.e., further adjusted for exercise for the association between $\mathrm{PM}_{2.5}$ exposure and death, or for $\mathrm{PM}_{2.5}$ exposure for the association between exercise and death). We performed a trend test across the exercise (i.e., inactive, moderate or high habitual exercise) and $\mathrm{PM}_{2.5}$ (i.e., low, moderate or high exposure) groups, respectively, with the corresponding group treated as an ordinal variable. To explore the overall interaction effect, we performed an additional test for interaction by including an interaction term in Model 2 that captured continuous $\mathrm{PM}_{2.5}$ (every $10 \mu \mathrm{g} / \mathrm{m}^{3}$ ) by exercise group.

We performed subgroup analyses for each $\mathrm{PM}_{2.5}$ and exercise group to evaluate the effects of $\mathrm{PM}_{2.5}$ exposure or habitual exercise in each stratum. Finally, we classified participants into 9 groups according to their $\mathrm{PM}_{2.5}$ exposure and habitual exercise; we used inactive participants with high $\mathrm{PM}_{2.5}$ exposure as the reference group.

We performed a series of sensitivity analyses to evaluate the robustness of our estimates. Firstly, we further adjusted for the presence of common chronic diseases, including diabetes (defined as fasting blood glucose $\geq 126 \mathrm{mg} / \mathrm{dL}$ or self-reported, physician-diagnosed diabetes), hypertension (defined as systolic blood pressure $\geq 140 \mathrm{~mm} \mathrm{Hg}$ or diastolic blood pressure $\geq 90 \mathrm{~mm} \mathrm{Hg}$, or self-reported, physician-diagnosed hypertension), dyslipidemia (defined as total cholesterol $\geq 240 \mathrm{mg} / \mathrm{dL}$, triglyceride $\geq 200 \mathrm{mg} / \mathrm{dL}$ or high-density lipoprotein cholesterol $<40 \mathrm{mg} / \mathrm{dL}$ ), cardiovascular disease (defined as self-reported, physiciandiagnosed coronary heart disease, stroke, peripheral arterial disease or aortic disease) and chronic obstructive pulmonary disease (defined as physician-diagnosed chronic obstructive pulmonary disease or a ratio of forced expiratory volume in $1 \mathrm{~s}$ to forced vital capacity $<70 \%$ ). In other sensitivity analyses, we used annual $\mathrm{PM}_{2.5}$ of the year of medical examination; we included participants who had partial data and used their previous or subsequent medical records to impute the missing values;-we restricted the analysis to the participants aged 65 years or older; we restricted the analysis to nonsmokers; we compared the effects on deaths from all causes (i.e., including deaths by accident, suicide and homicide, as well as natural causes) with the effects on death from all natural causes. Lastly, we analyzed only participants who gave a home (as opposed to work) address.

We conducted statistical analyses using software $R$ version 3.6.1. We treated the estimated effects as statistically significant if the 2 -tailed $p$ value $<0.05$.

\section{Ethics approval}

This study is approved by the Joint Chinese University of Hong Kong-New Territories East Cluster Clinical Research Ethics Committee (2018.388) and National Cheng Kung University in Tainan, Taiwan (A-ER-108-081).

\section{Results}

Table 1 shows the main characteristics of participants, all observations and deaths from natural causes. We included a total of 384130 adults ( $\geq 18 \mathrm{yr}$ ), with 842394 medical examination records between 2001 and 2016, in the main analysis. The median number of medical visits during the study period was 1.0, with an interquartile range (IQR) of 1.0-3.0. The median visit interval was 3.3 years, with an IQR of $1.7-$ 9.5 years. The median duration of follow-up was 13.4 years, with an IQR of 9.4-16.4 years. We followed participants for a total of 4.9 million person-years. Information on medical examinations by the 9 exercise and $\mathrm{PM}_{2.5}$ groups are shown in Appendix 1, Table E1. We identified 12375 natural cause deaths during the study period, for an incidence rate of 2.5 per 1000 person-years. Figure 1 shows the distribution of $\mathrm{PM}_{2.5}$ concentrations and participants by year. Concentrations of $\mathrm{PM}_{2.5}$ (overall IQR was $21.6-27.8 \mu \mathrm{g} / \mathrm{m}^{3}$ ) varied widely across the island (Figure 2).

The main effects of habitual exercise and $\mathrm{PM}_{2.5}$ on risk of death are shown in Table 2. A higher level of habitual exercise was associated with a lower risk of death. In contrast, a higher level of $\mathrm{PM}_{2.5}$ exposure was associated with a higher risk of death. Mutual adjustment for $\mathrm{PM}_{2.5}$ exposure and exercise did not substantially change the associations. We observed significant trends for the associations. The interaction test showed a minor, but statistically significant, interaction effect of exercise and $\mathrm{PM}_{2.5}$ on the risk of death (HR 1.03, 95\% Cl 1.01-1.06).

In subgroup analyses, habitual exercise was associated with a lower risk of death in each stratum of $\mathrm{PM}_{2.5}$ exposure, whereas $\mathrm{PM}_{2.5}$ exposure was associated with a higher risk of death in each exercise stratum (except for the inactive stratum) (Table 3).

Figure 3 shows the combined effects of exercise and $\mathrm{PM}_{2.5}$ exposure on risk of death. Participants with a high level of exercise and an exposure to low $\mathrm{PM}_{2.5}$ concentrations had the lowest risk of death ( $\mathrm{HR} 0.54,95 \% \mathrm{Cl} 0.50-0.58$ ), and inactive participants with an exposure to high $\mathrm{PM}_{2.5}$ concentrations had the highest risk of death from natural causes. Risk of death and $\mathrm{PM}_{2.5}$ and were positively associated, except for participants who were inactive. Dose-response associations between exercise and risk of death were generally evident for participants exposed to different levels of $\mathrm{PM}_{2.5}$ air pollution.

Sensitivity analyses yielded similar results (Appendix 1, Tables E2-E8). The HRs of $\mathrm{PM}_{2.5}$ exposures were slightly greater in participants who had higher levels of exercise, and we observed weak interactions between $\mathrm{PM}_{2.5}$ and exercise.

\section{Interpretation}

We investigated the combined effects of long-term exposure to ambient $\mathrm{PM}_{2.5}$ and habitual exercise on the risk of death from natural causes in a large, longitudinal cohort that enabled us to have sufficient power to obtain stable and precise estimates and to conduct subgroup and sensitivity analyses to test the robustness of the associations and identify sensitive subpopulations. 
Table 1: Participant characteristics

\section{Characteristics}

Age, yr, mean \pm SD

Sex, male

Education

Lower than high school

High school

College or university

Postgraduate

Cigarette smoking

Never

Former

Current

Alcohol consumption

Never or seldom

Former

Current

Physical labour at work

Mostly sedentary

Sedentary with occasional walking

Mostly standing or walking

Hard labour

Habitual exercise group $\dagger$

Inactive

Moderate

High

Exercise, MET-h, mean \pm SD

Vegetable intake

Seldom

Moderate

Frequent

Fruit intake

Seldom

Moderate

Frequent

Occupational exposure

Body mass index $\left(\mathrm{kg} / \mathrm{m}^{2}\right)$, mean $\pm \mathrm{SD}$

$\mathrm{PM}_{2.5}\left(\mu \mathrm{g} / \mathrm{m}^{3}\right) \ddagger$, mean $\pm \mathrm{SD}$

$\mathrm{PM}_{2.5}$ by exercise groupt, mean $\pm \mathrm{SD}$

Inactive

Moderate

High
No. (\%) of patients at

$$
\text { baseline* }
$$

$n=384130$

$39.2 \pm 12.7$

$186985(48.7)$

$56862(14.8)$

75248 (19.6)

$204730(53.3)$

47290 (12.3)

$284311(74.0)$

$21944(5.7)$

77875 (20.3)

$330179(86.0)$

36063 (9.4)

$17888(4.7)$

242748 (63.2)

$100314(26.1)$

$33123(8.6)$

$7945(2.1)$

173948 (45.3)

113669 (29.6)

96513 (25.1)

$7.2 \pm 13.0$

54200 (14.1)

226735 (59.0)

$103195(26.9)$

132705 (34.5)

206117 (53.7)

45308 (11.8)

$31146(8.1)$

$23.0 \pm 3.7$

$26.4 \pm 7.6$

$26.6 \pm 7.6$

$26.3 \pm 7.5$

$26.2 \pm 7.6$
No. (\%) of deaths from

natural causes (mortality

No. (\%) of observations *

$n=842394$

rate, per 1000 person-years) $n=12375$ (2.5)

$41.6 \pm 12.5$

424495 (50.4)

7206 (3.1)

110341 (13.1)

7014 (9.4)

159295 (18.9)

459089 (54.5)

113669 (13.5)

$2268(2.3)$

$2753(1.1)$

$340(0.6)$

635672 (75.5)

$7671(2.1)$

$50776(6.0)$

1361 (5.1)

155946 (18.5)

$3343(3.4)$

720674 (85.6)

$10002(2.4)$

$82329(9.8)$

$39391(4.7)$

$1048(2.3)$

$1325(5.9)$

556806 (66.1)

7559 (2.5)

208090 (24.7)

3095 (2.4)

$63685(7.6)$

1372 (3.3)

$13813(1.6)$

$349(3.5)$

323617 (38.4)

5290 (2.3)

266415 (31.6)

$1904(2.3)$

252362 (30.0)

5181 (3.0)

$8.5 \pm 14.2$

99349 (11.8)

2005 (2.9)

493825 (58.6)

$7083(2.5)$

3287 (2.6)

249220 (29.6)

4164 (2.6)

$6747(2.6)$

1464 (2.5)

745 (1.9)

$23.2 \pm 3.6$

$26.3 \pm 7.4$

$26.6 \pm 7.5$

$26.2 \pm 7.4$

$26.0 \pm 7.4$

Note: $\mathrm{MET}$ = metabolic equivalent, PM2.5 = fine particle matter, $\mathrm{SD}$ = standard deviation.

*Unless indicated otherwise.

†The tertile cut-off points for exercise volume using the MET value and duration (h): inactive (0 MET-h), moderate ( 0 to $8.75 \mathrm{MET}$-h) and high (> 8.75 MET-h). The tertile cut-off points for $\mathrm{PM}_{25}$ : low $\left(<22.4 \mu \mathrm{g} / \mathrm{m}^{3}\right)$, moderate $\left(22.4\right.$ to $\left.26.0 \mu \mathrm{g} / \mathrm{m}^{3}\right)$ and high $\left(\geq 26.0 \mu \mathrm{g} / \mathrm{m}^{3}\right)$.

FRefers to the 2-year average $\mathrm{PM}_{25}$ concentration in the year of medical visit and in the year before the medical visit. 
The longitudinal, open study design allowed for recruitment of a large sample and for the study of changing $\mathrm{PM}_{2.5}$ exposure combined with exercise over time. Our results show that high levels of habitual exercise and low levels of $\mathrm{PM}_{2.5}$ exposure are associated with lowest risk of death and that habitual exercise reduced the risk of death across $\mathrm{PM}_{2.5}$ categories, compared to inactivity. We observed weak interaction effects between $\mathrm{PM}_{2.5}$ and exercise on risk of death.

Previous studies have reported similar associations between air pollution and risk of death. ${ }^{22-24}$ The association between risk of death and air pollution found in this study (HR 1.18 per $10 \mu \mathrm{g} / \mathrm{m}^{3}$ ) was slightly larger than those found in Europe (HR 1.02 per $\left.10 \mu \mathrm{g} / \mathrm{m}^{3}\right)^{23}$ and in the United States (HR 1.07 per $\left.10 \mu \mathrm{g} / \mathrm{m}^{3}\right)^{24}$ possibly because of higher exposures in our cohort. Moreover, we considered clustering effects within the same city, and thus included a city-level random intercept in our analysis, which is known to result in larger HRs. ${ }^{25}$

In line with the benefits of habitual exercise previously documented, ${ }^{13,26}$ we identified an inverse association between habitual exercise and risk of death. However, it is difficult to compare the magnitudes of the direct benefits of exercise. Some previous studies did not find a threshold for the benefits of exercise, but showed that even a low level of exercise can benefit human health. ${ }^{13,27,28}$

Unlike most previous studies that used modelling methods based on literature-derived risks of air pollution and benefits of exercise, ${ }^{29-32}$ this study provided evidence of the effects of habitual exercise on the risk of death in a population exposed to different levels of $\mathrm{PM}_{2.5}$ that were directly measured. The information we collected on physical activity was comprehensive, including various types of exercise and physical activity during leisure time and daily work. Three previous cohort studies targeting older adults ${ }^{8,9}$ and women $^{10}$ have been conducted in Hong Kong, ${ }^{8}$ Denmark ${ }^{9}$ and the US. ${ }^{10}$ The Hong Kong study had a similar air pollution level to that of our study, and the studies in Denmark and the US had better air quality. However, the results from our study and all 3 of these studies suggest that habitual exercise is beneficial, even for people living in relatively polluted regions. Unlike previous studies, we observed a weak, but statistically significant, interaction between habitual exercise and $\mathrm{PM}_{2.5}$ exposure, probably because of the large sample size or the different population in our study. We did not observe a significant interaction effect in the sensitivity analysis that included only participants aged 65 years and older (Appendix 1, Table E5).

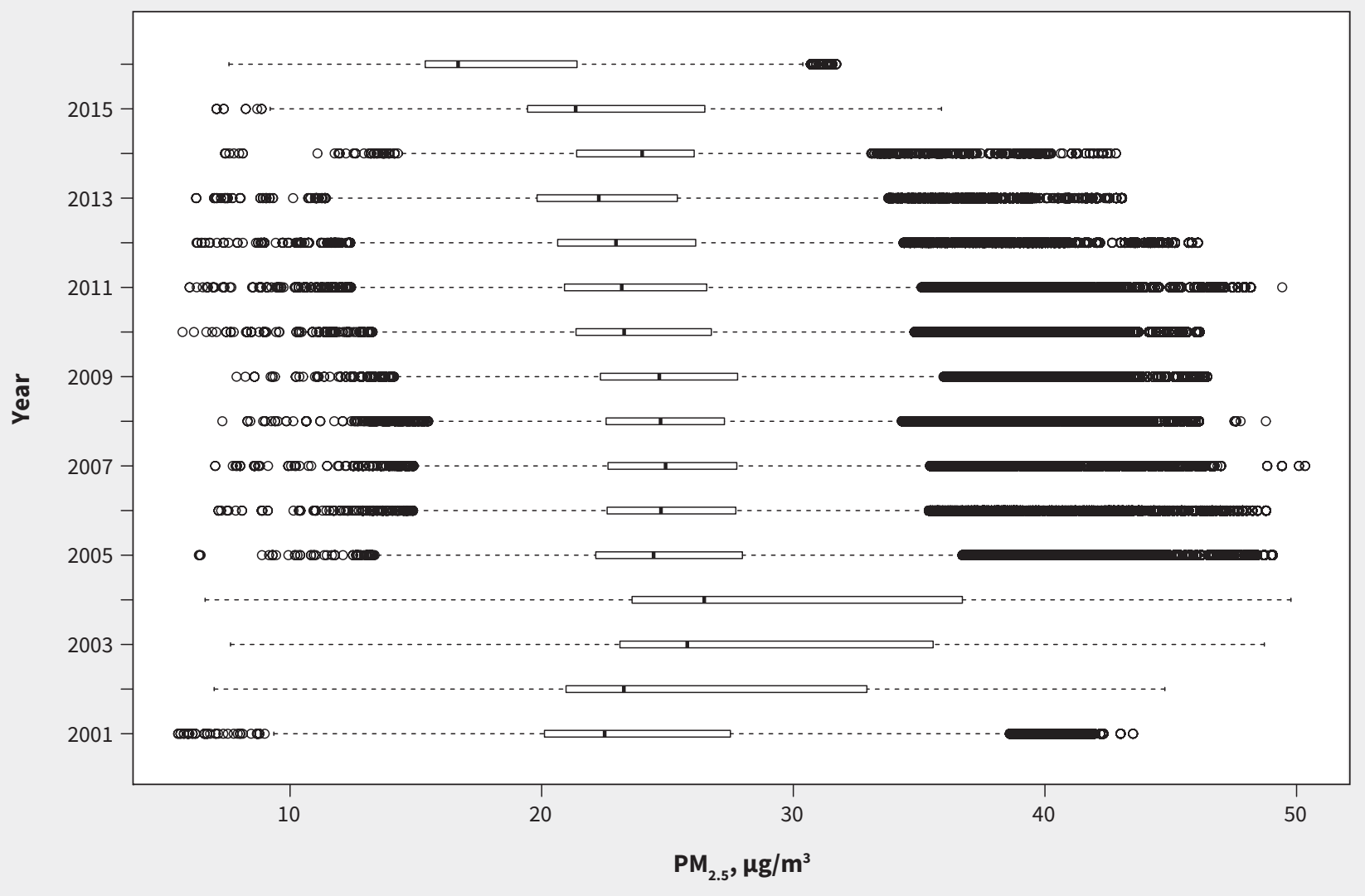

Figure 1: The temporal distribution of the 2-year average air pollution (measured by concentration of fine particle matter, $\mathrm{PM}_{2.5}$ ) by year for the 842394 medical visits of the 384130 participants in Taiwan. Boxes represent the interquartile range (IQR), with centre lines showing the median concentration. Whiskers extend to the highest observations within 3 IQRs of the box, with more extreme observations shown as circles. 
The results of our study are also in line with those of most previous studies showing that exercise has benefits for people in polluted areas in the context of other health outcomes, including hypertension, ${ }^{33}$ diabetes, ${ }^{34}$ systemic inflammation, ${ }^{35}$ myocardial infarction, ${ }^{36}$ lung function, ${ }^{20}$ stroke ${ }^{37}$ and asthma. ${ }^{38}$ Although statistically significant interactions have been reported for lung function, stroke and asthma, ${ }^{20,37,38}$ the interaction strengths are generally weak, which is similar to the results of our study. More research is warranted to explore the combined effects on different health outcomes and potential interactions.
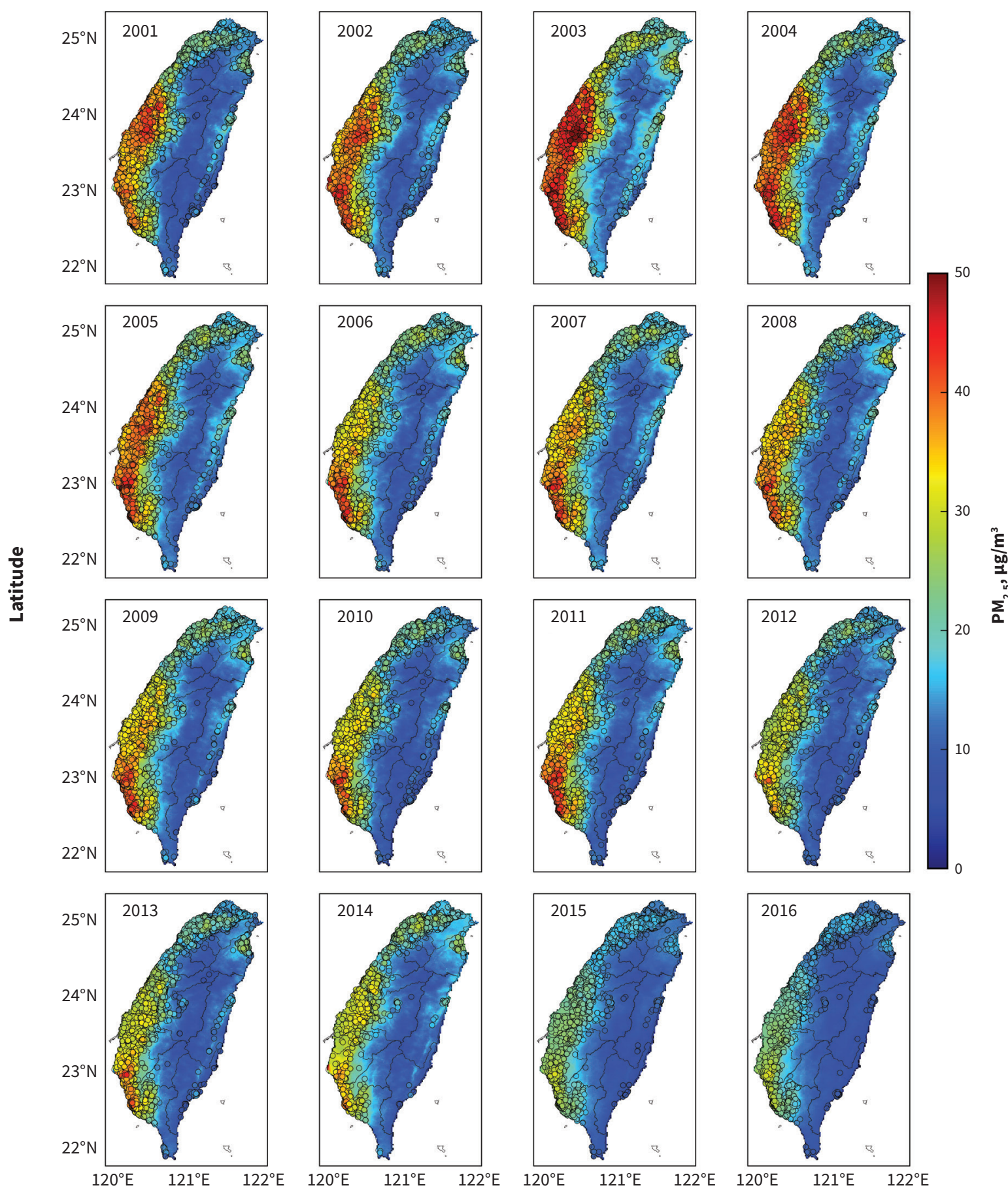

\section{Longitude}

Figure 2: The spatial distribution of participants and air pollution (measured by concentration of fine particle matter, $\mathrm{PM}_{2.5}$ ) in Taiwan. Circles indicate the locations of participants. 
Table 2: Associations of death from natural causes with habitual exercise and exposure to air pollution (PM 2.5$)$ among adults in Taiwan*

\begin{tabular}{|c|c|c|c|c|}
\hline Modelt & Hazard ratio & $p$ value & Hazard ratioł & $p$ value \\
\hline \multicolumn{5}{|l|}{ Main effects of exercise } \\
\hline \multicolumn{5}{|l|}{ Model 1} \\
\hline Inactive & Reference & - & Reference & - \\
\hline Moderate exercise & $0.79(0.75-0.82)$ & $<0.001$ & $0.79(0.75-0.82)$ & $<0.001$ \\
\hline High exercise & $0.59(0.56-0.61)$ & $<0.001$ & $0.59(0.57-0.62)$ & $<0.001$ \\
\hline Test for trend & $0.77(0.75-0.78)$ & $<0.001$ & $0.77(0.75-0.79)$ & $<0.001$ \\
\hline \multicolumn{5}{|l|}{ Model 2} \\
\hline Inactive & Reference & - & Reference & - \\
\hline Moderate exercise & $0.84(0.80-0.88)$ & $<0.001$ & $0.84(0.80-0.88)$ & $<0.001$ \\
\hline High exercise & $0.65(0.62-0.68)$ & $<0.001$ & $0.65(0.62-0.68)$ & $<0.001$ \\
\hline Test for trend & $0.81(0.79-0.82)$ & $<0.001$ & $0.81(0.79-0.83)$ & $<0.001$ \\
\hline \multicolumn{5}{|l|}{ Main effects of $\mathrm{PM}_{2.5}$ exposure } \\
\hline \multicolumn{5}{|l|}{ Model 1} \\
\hline Low $\mathrm{PM}_{2.5}$ & Reference & - & Reference & - \\
\hline Moderate $\mathrm{PM}_{2.5}$ & $1.03(0.99-1.08)$ & 0.1 & $1.02(0.97-1.06)$ & 0.5 \\
\hline High $\mathrm{PM}_{2.5}$ & $1.14(1.09-1.19)$ & $<0.001$ & $1.13(1.08-1.18)$ & $<0.001$ \\
\hline Test for trend & $1.07(1.05-1.09)$ & $<0.001$ & $1.06(1.04-1.09)$ & $<0.001$ \\
\hline Per $10 \mu \mathrm{g} / \mathrm{m}^{3}$ of exposure & $1.26(1.20-1.31)$ & $<0.001$ & $1.21(1.16-1.26)$ & $<0.001$ \\
\hline \multicolumn{5}{|l|}{ Model 2} \\
\hline Low $\mathrm{PM}_{2.5}$ & Reference & - & Reference & - \\
\hline Moderate $\mathrm{PM}_{2.5}$ & $1.04(0.99-1.08)$ & 0.1 & $1.02(0.98-1.07)$ & 0.4 \\
\hline High $\mathrm{PM}_{2.5}$ & $1.17(1.12-1.22)$ & $<0.001$ & $1.15(1.10-1.20)$ & $<0.001$ \\
\hline Test for trend & $1.08(1.06-1.10)$ & $<0.001$ & $1.07(1.04-1.09)$ & $<0.001$ \\
\hline Per $10 \mu \mathrm{g} / \mathrm{m}^{3}$ of exposure & $1.22(1.17-1.27)$ & $<0.001$ & $1.18(1.14-1.23)$ & $<0.001$ \\
\hline
\end{tabular}

Table 3: Associations of death from natural causes with habitual exercise and exposure to air pollution $\left(\mathrm{PM}_{2.5}\right)$ among adults in Taiwan, stratified by categories of $\mathrm{PM}_{2,5}$ or habitual exercise*

\begin{tabular}{|c|c|c|c|c|c|c|}
\hline Model & Hazard ratio & $p$ value & Hazard ratio & $p$ value & Hazard ratio & $p$ value \\
\hline Stratified by $\mathrm{PM}_{2.5}$ & \multicolumn{2}{|c|}{ Low $\mathrm{PM}_{2.5}$} & \multicolumn{2}{|c|}{ Moderate $\mathrm{PM}_{2.5}$} & \multicolumn{2}{|c|}{ High PM $_{2.5}$} \\
\hline Inactive & Reference & - & Reference & - & Reference & - \\
\hline Moderate exercise & $0.81(0.74-0.87)$ & $<0.001$ & $0.87(0.81-0.95)$ & 0.001 & $0.84(0.78-0.90)$ & $<0.001$ \\
\hline High exercise & $0.59(0.55-0.64)$ & $<0.001$ & $0.71(0.65-0.77)$ & $<0.001$ & $0.67(0.62-0.72)$ & $<0.001$ \\
\hline Test for trend & $0.77(0.74-0.80)$ & $<0.001$ & $0.84(0.81-0.88)$ & $<0.001$ & $0.82(0.79-0.85)$ & $<0.001$ \\
\hline Stratified by exercise & \multicolumn{2}{|c|}{ Inactive } & \multicolumn{2}{|c|}{ Moderate exercise } & \multicolumn{2}{|c|}{ High exercise } \\
\hline Low $\mathrm{PM}_{2.5}$ & Reference & - & Reference & - & Reference & - \\
\hline Moderate $\mathrm{PM}_{2.5}$ & $0.93(0.86-1.00)$ & 0.04 & $1.02(0.94-1.11)$ & 0.7 & $1.15(1.07-1.25)$ & $<0.001$ \\
\hline High $\mathrm{PM}_{2.5}$ & $0.99(0.91-1.07)$ & 0.7 & $1.13(1.03-1.24)$ & 0.008 & $1.34(1.22-1.47)$ & $<0.001$ \\
\hline Test for trend & $0.98(0.94-1.02)$ & 0.3 & $1.06(1.01-1.11)$ & 0.01 & $1.16(1.11-1.21)$ & $<0.001$ \\
\hline Per $10 \mu \mathrm{g} / \mathrm{m}^{3}$ & $1.06(1.01-1.11)$ & 0.02 & $1.10(1.04-1.16)$ & 0.001 & $1.44(1.34-1.55)$ & $<0.001$ \\
\hline
\end{tabular}

*The models fully adjusted for age, sex, educational level, body mass index, physical labour at work, cigarette smoking, alcohol drinking, vegetable intake, fruit intake, occupational exposure, season, and year of enrolment. The tertile cut-off points for exercise volume using the metabolic equivalent (MET) value and duration ( $\mathrm{h}$ ): inactive (MET- $\mathrm{h}=0)$, moderate $(M E T-h=0$ to 8.75$)$ and high (MET-h $>8.75)$. The tertile cut-off points for $\mathrm{PM}_{2.5}$ : low $\left(<22.4 \mu \mathrm{g} / \mathrm{m}^{3}\right)$, moderate $\left(22.4 \mathrm{to} 26.0 \mu \mathrm{g} / \mathrm{m}^{3}\right)$ and high $\left(\geq 26.0 \mu \mathrm{g} / \mathrm{m}^{3}\right)$. 

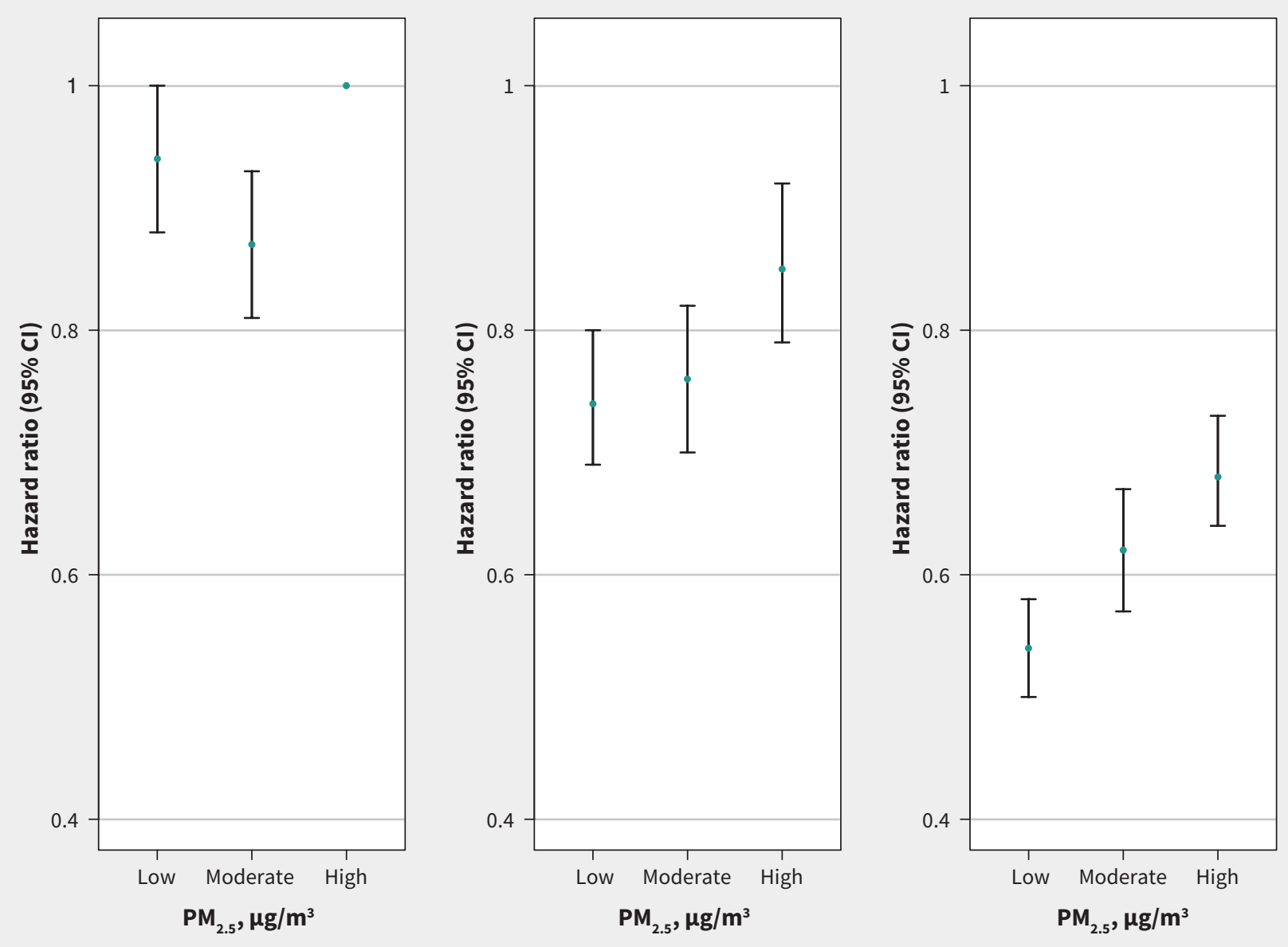

Figure 3: Combined effects of habitual exercise and air pollution (measured by the concentration of fine particle matter, $\mathrm{PM}_{2.5}$ ) on risk of death in adults in Taiwan. Hazard ratios (HRs) with $95 \%$ confidence intervals (Cls) show the association of low, moderate or high $\mathrm{PM}_{2.5}$ exposure and risk of death among-participants who were (A) inactive, or with (B) moderate or (C) high levels of habitual exercise. Models adjusted for age, sex, education, body mass index, physical labour at work, cigarette smoking, alcohol consumption, vegetable and fruit intake, occupational exposure to dust and solvents, season and year of cohort enrolment. We treated the group of inactive participants with high $\mathrm{PM}_{2.5}$ exposure as the reference group. The tertile cut-off points for exercise volume, using the metabolic equivalent (MET) value and duration (h): inactive (0 MET-h), moderate (0 to $8.75 \mathrm{MET}-\mathrm{h})$ and high (>8.75 MET-h). The tertile cut-off points for $\mathrm{PM}_{2.5}$ : low $\left(<22.4 \mu \mathrm{g} / \mathrm{m}^{3}\right)$, moderate $\left(22.4\right.$ to $\left.26.0 \mu \mathrm{g} / \mathrm{m}^{3}\right)$ and high $\left(\geq 26.0 \mu \mathrm{g} / \mathrm{m}^{3}\right)$.

\section{Limitations}

We did not distinguish between indoor or outdoor habitual exercise. However, $92.7 \%$ of Taiwanese residents reported that they preferred outdoor exercise in a national survey in $2017 .{ }^{39}$ We used the estimated $\mathrm{PM}_{2.5}$ concentrations at participant addresses to indicate the level of exposure during exercise. Although the variation in $\mathrm{PM}_{2.5}$ concentrations within a certain area is generally small and most Taiwanese residents have been reported to undertake exercise near their homes, ${ }^{39}$ it was difficult to avoid random exposure misclassification, which might attenuate the estimated associations. Similarly, we used the 2-year average concentration as a surrogate measure of $\mathrm{PM}_{2.5}$ exposure, which might not be the exact level of exposure during exercise. More advanced technologies are needed for accurate assessment of individual exposure in future studies. Fourth, some participants may have been lost to follow-up if they left Taiwan during the study period. However, only $0.16 \%-0.28 \%$ of people in Taiwan migrated each year during the study period. ${ }^{40}$ Therefore, emigration is not expected to bias our main findings. A common limitation of exercise studies is that healthier participants may undertake higher levels of physical activity, and those with health problems may undertake lower levels of physical activity. However, the sensitivity analysis adjusting for the presence of common chronic diseases showed results consistent with our main findings. Participants were enrolled through a paid membership and had relatively high levels of education and economic status, and our study was conducted in a moderately polluted area. Therefore, generalizing results to other populations and regions should be done with caution.

\section{Conclusion}

We found that a high level of habitual exercise and a low level of exposure to air pollution was associated with lower risk of death from natural causes, whereas a low level of habitual exercise and 
a high level of exposure was associated with higher risk of death. Habitual exercise reduces the risk of death regardless of exposure to air pollution, and air pollution generally increases the risk of death regardless of habitual exercise. Thus, habitual exercise should be promoted as a health improvement strategy, even for people residing in relatively polluted areas.

\section{References}

1. Giles-Corti B, Vernez-Moudon A, Reis R, et al. City planning and population health: a global challenge. Lancet 2016;388:2912-24.

2. GBD Compare Data Visualization. Seattle: Institute for Health Metrics and Evaluation, University of Washington, 2020. Available: http://vizhub.healthdata. org/gbd-compare (accessed 2021 Jun. 23)

3. Ambient (outdoor) air pollution. Geneva: World Health Organization; 2018. Available: https://www.who.int/news-room/fact-sheets/detail/ambient-(outdoor) -air-quality-and-health (accessed 2020 Jan. 3).

4. Lee I-M, Shiroma EJ, Lobelo F, et al. Effect of physical inactivity on major noncommunicable diseases worldwide: an analysis of burden of disease and life expectancy. Lancet 2012;380:219-29.

5. Global action plan on physical activity 2018-2030. Geneva: World Health Organization; 2018. Available: https://apps.who.int/iris/bitstream/handle/10665/ 272722/9789241514187-eng.pdf(accessed 2020 Apr. 12).

6. Sinharay R, Gong J, Barratt B, et al. Respiratory and cardiovascular responses to walking down a traffic-polluted road compared with walking in a traffic-free area in participants aged 60 years and older with chronic lung or heart disease and age-matched healthy controls: a randomised, crossover study. Lancet 2018; 391:339-49.

7. McCreanor J, Cullinan P, Nieuwenhuijsen MJ, et al. Respiratory effects of exposure to diesel traffic in persons with asthma. N Engl J Med 2007;357: 2348-58.

8. Sun S, Cao W, Qiu H, et al. Benefits of physical activity not affected by air pollution: a prospective cohort study. Int J Epidemiol 2020;49:142-52.

9. Andersen ZJ, de Nazelle A, Mendez MA, et al. A study of the combined effects of physical activity and air pollution on mortality in elderly urban residents: the Danish diet, cancer, and health cohort. Environ Health Perspect 2015;123: 557-63.

10. Elliott $E G$, Laden $F$, James $P$, et al. Interaction between long-term exposure to fine particulate matter and physical activity, and risk of cardiovascular disease and overall mortality in US women. Environ Health Perspect 2020;128:127012.

11. Chu H-J, Ali MZ, He Y-C. Spatial calibration and PM ${ }_{25}$ mapping of low-cost air quality sensors. Sci Rep 2020;10:1-11.

12. Guo C, Zhang Z, Lau AKH, et al. Effect of long-term exposure to fine particulate matter on lung function decline and risk of chronic obstructive pulmonary disease in Taiwan: a longitudinal, cohort study. Lancet Planet Health 2018;2: e114-e125.

13. Wen CP, Wai JPM, Tsai MK, et al. Minimum amount of physical activity for reduced mortality and extended life expectancy: a prospective cohort study. Lancet 2011;378:1244-53.

14. The introduction of MJ Health Database: technical report no. MJHRF-TR-01. Taipei (Taiwan): MJ Health Research Foundation; 2016.

15. Cause of death data. Taipei: Ministry of Health and Welfare; 2019. Available: https://www.mohw.gov.tw/mp-1.html (accessed 22 Mar. 2021).

16. Lin C, Li Y, Yuan Z, et al. Using satellite remote sensing data to estimate the high-resolution distribution of ground-level $\mathrm{PM}_{2.5}$. Remote Sens Environ 2015; 156:117-28.

17. Zhang Z, Chang L-Y, Lau AK, et al. Satellite-based estimates of long-term exposure to fine particulate matter are associated with C-reactive protein in 30034 Taiwanese adults. Int J Epidemiol 2017; 46:1126-36.

18. Lao XQ, Deng H-B, Liu X, et al. Increased leisure-time physical activity associated with lower onset of diabetes in 44828 adults with impaired fasting glucose: a population-based prospective cohort study. Br J Sports Med 2018; 53:895-900.
19. Guo C, Tam T, Bo Y, et al. Habitual physical activity, renal function and chronic kidney disease: A cohort study of nearly 200,000 adults. Br J Sports Med 2020;54: 1225-30.

20. Guo $C, B$, Y, Chan T-C, et al. Does fine particulate matter $\left(\mathrm{PM}_{2.5}\right)$ affect the benefits of habitual physical activity on lung function in adults: a longitudinal cohort study. BMC Med 2020;18:134.

21. Ainsworth BE, Haskell WL, Whitt MC, et al. Compendium of physical activities: an update of activity codes and MET intensities. Med Sci Sports Exerc 2000;32: S498-504.

22. Liu C, Chen R, Sera F, et al. Ambient particulate air pollution and daily mortality in 652 cities. N Engl J Med 2019;381:705-15.

23. Carey IM, Atkinson RW, Kent AJ, et al. Mortality associations with long-term exposure to outdoor air pollution in a national English cohort. Am J Respir Crit Care Med 2013;187:1226-33.

24. Di Q, Wang $\mathrm{Y}$, Zanobetti A, et al. Air pollution and mortality in the medicare population. N Engl J Med 2017;376:2513-22.

25. Beelen R, Raaschou-Nielsen $\mathrm{O}$, Stafoggia $\mathrm{M}$, et al. Effects of long-term exposure to air pollution on natural-cause mortality: an analysis of 22 European cohorts within the multicentre ESCAPE project. Lancet 2014;383:785-95.

26. Gebel K, Ding D, Chey T, et al. Effect of moderate to vigorous physical activity on all-cause mortality in middle-aged and older Australians. JAMA Intern Med 2015;175:970-7.

27. Ekelund U, Tarp J, Steene-Johannessen J, et al. Dose-response associations between accelerometry measured physical activity and sedentary time and all cause mortality: systematic review and harmonised meta-analysis. BMJ 2019;366:14570.

28. Arem H, Moore SC, Patel A, et al. Leisure time physical activity and mortality: a detailed pooled analysis of the dose-response relationship. JAMA Intern Med 2015;175:959-67.

29. Hankey S, Marshall JD, Brauer M. Health impacts of the built environment: within-urban variability in physical inactivity, air pollution, and ischemic heart disease mortality. Environ Health Perspect 2012;120:247-53.

30. Cepeda M, Schoufour J, Freak-Poli R, et al. Levels of ambient air pollution according to mode of transport: a systematic review. Lancet Public Health 2017;2:e23-34.

31. Johan de Hartog JJ, Boogaard H, Nijland H, et al. Do the health benefits of cycling outweigh the risks? Environ Health Perspect 2010;118:1109.

32. Rojas-Rueda D, de Nazelle A, Tainio M, et al. The health risks and benefits of cycling in urban environments compared with car use: health impact assessment study. BMJ 2011;343:d4521.

33. Guo C, Zeng Y, Chang L, et al. Independent and opposing associations of habitual exercise and chronic $\mathrm{PM}_{2.5}$ exposures on hypertension incidence. Circulation 2020;142:645-56.

34. Guo C, Yang HT, Chang L, et al. Habitual exercise is associated with reduced risk of diabetes regardless of air pollution: a longitudinal cohort study. Diabetologia 2021;64:1298-1308.

35. Zhang Z, Hoek G, Chang L, et al. Particulate matter air pollution, physical activity and systemic inflammation in Taiwanese adults. Int J Hyg Environ Health 2018;221:41-7.

36. Kubesch NJ, Therming Jørgensen J, Hoffmann B, et al. Effects of leisure time and transport-related physical activities on the risk of incident and recurrent myocardial infarction and interaction with traffic-related air pollution: a cohort study. J Am Heart Assoc 2018;7:e009554.

37. Lin $\mathrm{H}, \mathrm{Guo} \mathrm{Y}$, Di Q, et al. Ambient $\mathrm{PM}_{25}$ and stroke: effect modifiers and population attributable risk in six low- and middle-income countries. Stroke 2017;48:1191-7.

38. McConnell R, Berhane K, Gilliland F, et al. Asthma in exercising children exposed to ozone: a cohort study. Lancet 2002;359:386-91.

39. Report of active cities, Taiwan. Taiwan: Department of Physical Education Ministry of Education; 2017. Available: https://isports.sa.gov.tw/apps/Download. aspx?SYS=TIS\&MENU_CD=M07\&ITEM_CD=T01\&MENU_PRG_CD=4\&ITEM_PRG_CD $=2$ (accessed 2021 Jun. 23).

40. Statistical yearbook of interior, 2021. Taiwan: Ministry of the Interior; 2021. Available: https://statis.moi.gov.tw/micst/stmain.jsp?sys=100 (accessed 2021 Apr. 20). 
Competing interests: None declared.

This article has been peer reviewed.

Affiliations: Jockey Club School of Public Health and Primary Care (Guo, Yang, Bo, Zeng, Lao), the Chinese University of Hong Kong, Hong Kong SAR, China; Department of Public Health (Yu), National Cheng Kung University, Tainan, Taiwan; Institute of Sociology (Chang), Academia Sinica, Taipei, Taiwan; Division of Environment and Sustainability (Lin, Lau), the Hong Kong University of Science and Technology, Hong Kong SAR, China; Department of Civil and Environmental Engineering (Lau), the Hong Kong University of Science and Technology, Hong Kong SAR, China; Department of Nutrition and Food Hygiene (Bo), School of Public Health, Zhengzhou University, China; Department of Sociology (Tam), the Chinese University of Hong Kong, Hong Kong SAR, China; Shenzhen Research Institute of the Chinese University of Hong Kong (Lao), Shenzhen, China.

Contributors: Xiang Qian Lao conceived and designed the study. Ly-yun Chang, Alexis Lau, Tsung Yu, Tony Tam and Xiang Qian Lao acquired the data, which Cui Guo and Xiang Qian Lao analyzed and interpreted. Xiang Qian Lao and Cui Guo drafted the manuscript. All authors critically revised the manuscript, gave final approval of the version to be published and agreed to be accountable for all aspects of the work.

Content licence: This is an Open Access article distributed in accordance with the terms of the Creative Commons Attribution (CC BY-NC-ND 4.0) licence, which permits use, distribution and reproduction in any medium, provided that the original publication is properly cited, the use is noncommercial (i.e., research or educational use), and no modifications or adaptations are made. See: https://creativecommons.org/licenses/by-nc-nd/4.0/

Funding: This work was supported in part by the RGC-General Research Fund (14603019) and the Environmental Health Research Fund of the Chinese University of Hong Kong (7104946). Cui Guo is supported in part by the Faculty Postdoctoral Fellowship Scheme of the Faculty of Medicine of the Chinese University of Hong Kong. Yiqian Zeng is supported by the PhD Studentship of the Chinese University of Hong Kong.

Data sharing: All the data are available and accessible after appropriate request from the corresponding author.

Disclaimer: Any interpretation or conclusion related to this manuscript does not represent the views of MJ Health Research Foundation.

Acknowledgements: The authors acknowledge the MJ Health Research Foundation for the authorization of using MJ health data (authorization code: MJHR2019006A). They also thank the Health and Welfare Data Science Center, Ministry of Health and Welfare in Taiwan for the help with mortality data linkage.

Accepted: June 1, 2021

Correspondence to: Xiang Qian Lao, xqlao@cuhk.edu.hk 